\title{
Weight loss, adherence, and dietary intakes of free-living adults consuming calorie-restricted diets varying in macronutrient composition: the POUNDS LOST Study
}

\author{
Catherine Champagne ${ }^{1}$, Derek Miketinas ${ }^{2}$, Frank Sacks ${ }^{3}$ and George Bray ${ }^{1}$ \\ ${ }^{1}$ Pennington Biomedical Research Center, Louisiana State University, Baton Rouge, LA, USA, \\ ${ }^{2}$ Texas Woman's University, Houston, TX, USA and \\ ${ }^{3}$ Harvard T.H. Chan School of Public Health, Boston, MA, USA
}

\begin{abstract}
Various diets have been examined as potential weight-loss treatment for individuals with overweight and obesity. POUNDS LOST was a 2-year randomized clinical trial examining effects of four calorie-restricted diets of varying macronutrient composition on weight loss in free-living adults with obesity. The main results demonstrated that reducing calories, regardless of macronutrient content, can reduce body weight. Participants were randomized to receive one of four diets: 1) $20 \%$ fat, $15 \%$ protein; 2) $20 \%$ fat, $25 \%$ protein; 3) $40 \%$ fat, $15 \%$ protein; and 4) $40 \%$ fat, $25 \%$ protein. Eight hundred eleven (811) subjects participated in the trial and weight loss, as expected, varied considerably. Three hundred forty-five participants $(54.8 \%$ female; $86.5 \%$ Caucasian, $12.4 \%$ African American, and 1.1\% other) provided baseline and 6-month follow-up dietary data. Dietary adherence was defined as consuming within $+5 \%$ of the target macronutrient composition for fat and protein. Stepwise regression and cross-validation techniques were used to model weight loss as a function of the following predictors: age, dietary adherence, energy density change, and fiber intake. Additionally, we explored whether all four diet types were healthy using the Healthy Eating Index 2015 (USDA). At 6 months, participants assigned to each diet had lost an average of $6 \mathrm{~kg}$. Mean weight loss among adherers however was $-8.4+5.0 \mathrm{~kg}$ and also did not vary across diet-type. Change in energy density was positively associated with weight change while age, dietary adherence, and fiber intake were inversely associated with weight change. Fiber intake was the strongest predictor of weight change; on average, a $10 \mathrm{~g}$ increase in fiber intake was associated with $2.2 \mathrm{~kg}$ decrease in body weight after 6 months of the dietary intervention. Only $46(13.3 \%)$ of the 345 participants who provided follow-up data (61\% male, 93\% Caucasian) met the criteria for adherence to both energy $( \pm 10 \%$ of target) and macronutrient targets. A decrease in energy density was positively associated with weight change for each diet-type; however, this effect was most profound in the high-fat, average-protein diet. Healthy Eating Index 2015 scores for the diets consumed by the subjects were: LF/AP, $74.8 \pm 9.7$; LF/HP, $73.8 \pm 12.4$; HF/AP, $73.4 \pm 13.0$; and HF/HP, 74.2 \pm 9.8 , which exceeds the scores of most Americans. These results suggest that dietary factors may positively influence weight loss in addition to energy intake and macronutrient composition. Furthermore, modified macronutrient, calorie-reduced diets can indeed be healthy compared to current dietary habits of US adults.
\end{abstract}

\section{Conflict of Interest}

There is no conflict of interest. 